\title{
Special issue on Euler and Venn Diagrams: Guest Editors' introduction
}

Euler and Venn diagrams have been widely studied over recent years and their role in logical reasoning and information visualization is now prominent. Venn diagrams form the basis of what is widely regarded to be the first sound and complete diagrammatic logic, developed by Sun-Joo Shin in the 1990s. Since then, substantial research has been conducted on Euler and Venn diagrams, spanning logic, cognitive science and philosophy. The papers selected for this special issue encompass all these themes, bringing together a valuable collection of papers for researchers working in this area.

This special issue contains five papers, selected after an open call for papers. The submitted papers were typically reviewed by three anonymous reviewers with the top ranked papers that best fitted the theme of the special issue being accepted. The topics of the accepted papers cover the historical development of Venn diagrams, algorithms for the automated layout of Euler diagrams, an empirical study to analyse the way diagrams support reasoning tasks, a logic for reasoning with Venn diagrams using their Boolean interpretation, and automated theorem proving with an (Euler-based) spider diagram system. We are delighted the papers included in the special issue represent important and complementary contributions within a broad and inter-disciplinary scope.

The first contribution is on the representation of existential statements with Venn diagrams by Moktefi and Pietarinen. They argue that the existing commentary on the development of this type of notation tends to underestimate Venn's appreciation of the possibilities and problems of depicting existence or non-emptiness. Venn's own experiments with depicting existence are compared to those of other 19th Century pioneers of logical diagrams; his reluctance to include notation for depicting existence in his diagrams is shown to be based on philosophical rather than practical objections and the close correspondence between Venn diagrams and syllogistic reasoning. Modern approaches to depicting existence, absence, particular individuals and cardinality in diagrams is discussed.

The second contribution is by Schwartzentruber and concerns the generation of interactive Euler diagrams based on specifications in the Region Connection Calculus, and includes a description of a software tool based on the work. The diagrams are interactive because the expectation is that users of the software will rearrange automatically generated diagrams to produce more pleasing or appropriate layouts. Schwartzentruber explores three approaches to the problem: an algorithm based on constraint solving with local search, an algorithm inspired by the gradient method, which considers global rather than local goals, and, finally, a hybrid method combining the previous two. The complexity analysis shows that Schwartzentruber's improved local search and hybrid methods have better lower bounds than local search and gradient methods presented in the literature.

Next, Sato and Mineshima present an empirical investigation that analyses the factors that make a particular set visualisation support (or hinder) reasoning tasks. There is a wide-ranging discussion of the context to the question of the inferential efficacy of diagrams, which touches on historical topics and modern insights from disciplines such as cognitive science. They use syllogistic tasks as the focus of the investigations, in which participants judge the faithfulness of a visualisation to a sentence in natural language; the visualisations under consideration are Euler, Venn and linear diagrams. The findings of the study support the authors' hypothesis that Euler diagrams are effective not only in interpreting sentential premises but also in reasoning about semantic structures implicit in given sentences.

In the fourth contribution, Set Venn diagrams applied to inclusions and non-inclusions, De Freitas and Viana focus on the interpretation of Venn diagrams as "sets, and not as statements about sets", thus considering an alternative semantics to those typically defined. That is, a diagrammatic formula is a set of inclusions and noninclusions between Boolean literals. They formalise their system along these lines, including a set of inference rules which is shown to be sound and complete. Thus, this work is in line with the tradition begun by Shin and Hammer of investigating the formal properties of diagrammatic logics. However, the authors' approach of treating diagrams as terms suggests a new perspective on the correspondence between Venn diagrams and graph-theoretic results explored by the authors in other work.

Finally, Urbas, Stapleton and Jamnik present Speedith, their interactive theorem prover for reasoning with spider diagrams, representing the state-of-the-art in automated reasoning with diagrams. This paper includes a complete system description of Speedith, including the spider diagram logic and the software itself. The syntax and semantics of spider diagrams are presented, followed by a set of inference rules which are proved sound and complete. Next, the structure of the software, its reasoning engine and interface, and its use of the 
automated layout library iCircles, is given. A key aspect of Speedith's design is its ability to interact with other theorem provers, such as Isabelle, via a framework for heterogeneous reasoning. In this way, Speedith offers users the choice to reason diagrammatically when the context (the task in hand and the user's own cognitive preference) is appropriate.

We would like to thank the Editor-in-Chief Marcus Kracht and the Editorial Board of the Journal for supporting us with the organisation of this special issue. Furthermore, we thank the anonymous referees and an international Advisory Board formed for this special issue who assisted with the decisions on which papers to include. We are extremely grateful that all reviewers provided timely and comprehensive reviews of the papers. This ensured that only the best and most appropriate papers have been included in this special issue and allowed us to ensure timely publication. In addition, the referees' valuable comments have helped the authors improve their research contributions and, therefore, increased the quality of the papers accepted for inclusion. Finally, we thank all of the authors who submitted papers to this special issue.

Jim Burton and Gem Stapleton

Guest Editors

University of Brighton, UK 